\title{
Acute kidney injury (AKI): Risk assessment and management in hospital versus community
}

\author{
Kugananda Sri Paranthaman* and Muhammad Jawad \\ Acute Medical Unit, East Surrey Hospital, Surrey and Sussex Healthcare NHS Trust, UK
}

\begin{abstract}
A retrospective clinical audit undertaken at East Surrey Hospital looking at the risk assessment targeting modifiable risk factors and management of at-risk population of developing AKI in hospital versus community. The study was done over two 5 -week periods over the span of 2 years which included 100 patients. The results concluded that 2 of the highest risk factors of developing AKI in hospital were patient co-morbidities (55\%) and nephrotoxic medication (55\%) where else hypovolemia (52\%) was the biggest risk factor of developing AKI in the community. The results also noted overall better management of AKI in the community than in hospital, particularly regular nephrotoxic medication review (90\%), fluid balance monitoring (64\%), urine dip (75\%) and blood gas on admission (76\%). Advanced age (58\% above the age of 75) and previous history of AKI are common significant risk factors in developing AKI in both groups. The study led to an action plan of developing the hospital AKI guideline, with tailored focus on risk assessment and prevention, which included streamlining management of patients with AKI using an AKI care bundle as well as creating an AKI electronic alert to identify patients admitted with previous history of AKI
\end{abstract}

\section{Background}

$\mathrm{KI}$ is a syndrome characterized by the rapid loss of the kidney's excretory function and is typically diagnosed by rise in Creatinine $\geq$ $26 \mathrm{mmol}$ within 48 hours, overall rise in Creatinine over $50 \%$ of baseline in 7 days or fall in urine output $<0.5 \mathrm{ml} / \mathrm{kg} /$ hour for more than 6 hours. An underlying feature is a rapid decline in glomerular filtration rate (GFR) usually associated with decreases in renal blood flow [1]. Inflammation represents an important additional component of AKI leading to the extension phase of injury [2].

In terms of epidemiology, the main causes of AKI in urban areas of developing countries are hospital acquired (renal ischaemia, sepsis and nephrotoxic drugs) while in rural areas, it is more commonly a consequence of community acquired diseases (diarrhoea, dehydration, infectious diseases and animal venoms). Under-reporting of AKI especially in developing countries is also a major problem that relates with the true knowledge of its impact in many parts of the world.

In developed countries, the prevalence of AKI is increasing. In hospital inpatients, it is estimated to occur up to $15 \%$ and is more common in critically ill patients, in whom its prevalence is estimated to be up to $60 \%$. On the other hand, community AKI is usually uncommon although a recent study estimated its incidence at $4.3 \%$ among all hospital admissions. However, even this incidence remains an underestimate of the true impact of community acquired AKI due to non-referral of patients to hospitals [3].

AKI is associated with a high rate of adverse outcomes with mortality rates ranging between $25 \%$ to $80 \%$, depending on the cause and clinical status of the patient. These data highlight the importance of recognition and appropriate management of AKI, usually in collaboration with nephrologists and other subspecialists [4].

$\mathrm{AKI}$ is associated with short and long term adverse outcomes. In a systematic review of 24 studies that involved more than 71,000 patients, the mortality rate was $18.9 \%$ in the 'risk' class, $36.1 \%$ in the 'injury' class and $46.5 \%$ in the 'failure' class. In non-AKI patients, the overall mortality was $6.9 \%$. Among AKI patients, the relative risk for death in comparison to non-AKI patients was 2.40 for 'risk' class, 4.15 for 'injury' class and 6.15 for 'failure class.'

In terms of outcomes from AKI, various factors may influence both short- and long-term sequelae. One retrospective study demonstrates that chronic kidney disease (CKD) not only predisposes an individual to a higher risk of AKI but also longer duration of renal replacement therapy (RRT), and more in-hospital resuscitation was observed in this group. eGFR was an independent predictor of both 30-day mortality (hazard ratio (HR) 0.994, $95 \%$ confidence interval (CI) 0.990-0.998) and 1-year mortality (HR 0.996, 95 \% CI 0.993-1.000) [5] (Figure 1).

\section{Aim and Objectives}

The ultimate aim of this study was to identify population at risk (advanced age, diabetes, pre-existing CKD, Hypertension, Ischaemic Heart Disease and Peripheral Vascular Disease) of developing AKI as well as a holistic risk assessment targeting modifiable risk factors to minimize incidences of AKI, whether it be in hospital or in the community.

We also took the opportunity to develop a local guideline on the prevention, recognition and management of AKI as we identified a gap in AKI care within our hospital mainly due to the lack of local formal guidance to aid health care professionals in this acute setting.

${ }^{\star}$ Correspondence to: Kugananda Sri Paranthaman, Acute Medical Unit, East Surrey Hospital, Surrey and Sussex Healthcare NHS Trust, UK, E-mail: kugananda.sriparanthman@nhs.net

Key words: acute kidney injury, risk assessment, nephrotoxicity, renal ischaemia, sepsis

Received: July 20, 2020; Accepted: August 07, 2020; Published: August 11, 2020 


\section{RIFLE}

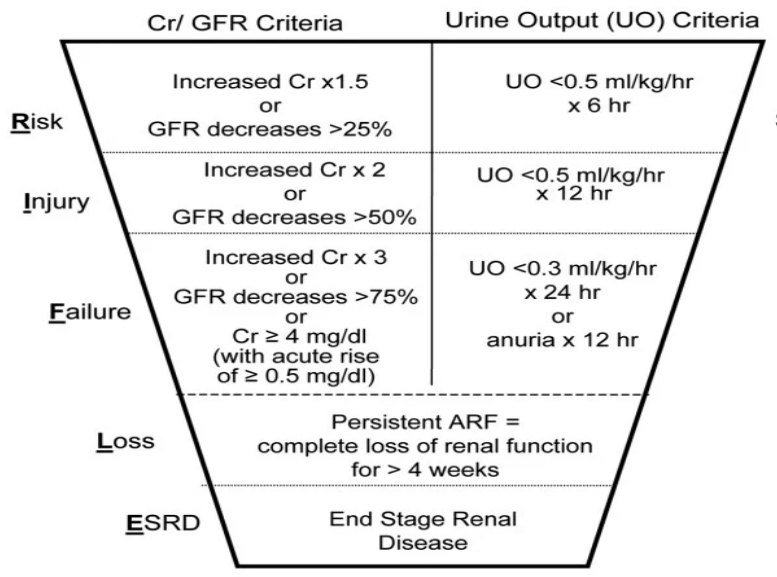

AKIN

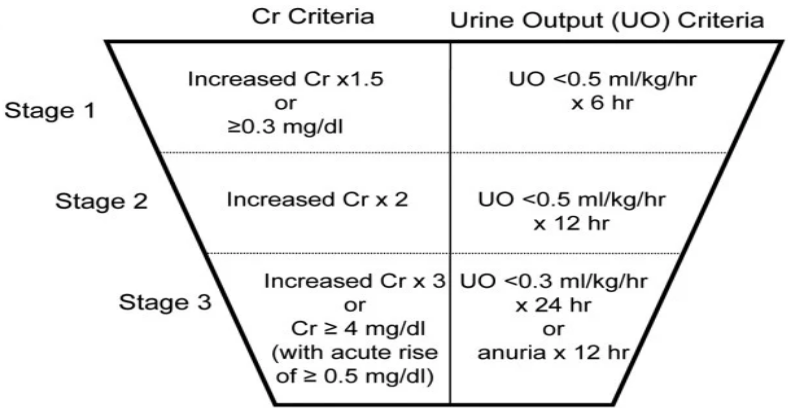

Patients who receive renal replacement therapy (RRT) are considered to have met the criteria for stage 3 irrespective of the stage that they are in at the time of commencement of RRT.

Figure 1. RIFLE and AKIN classifications of AKI

\section{Methodology}

The clinical audit consisted of 100 patients identified using the hospital AKI Alert System, which streamed patients into the inclusion group by alert notification of bloods of patients with significant rise in Creatinine and decline in eGFR in comparison to baseline. Data was collected over two 5-week periods over a period of 2 years. The patients were subsequently divided into two groups, those admitted with a community AKI (AKI on admission bloods) and those that developed AKI 24 hours after admission (AKI during hospital stay).

\section{Results}

The results of the clinical audit broadly looked at the risk factors involved in developing AKI and management of said patients, comparing those in hospital versus the community.

The most common risk factor for developing AKI within both groups was a non-modifiable risk factor, age $>75$ (58\%). Following this, within the hospital AKI group, the most common risk factors in descending order were nephrotoxics (55\%), chronic diseases (55\%) and deteriorating NEWS score (53\%). $40 \%$ of patients within the hospital AKI group had a previously documented AKI. In the community AKI group, the most common risk factors involved in descending order were hypovolaemia (52\%), CKD (43\%) and nephrotoxics (38\%).

In regards to management, within the hospital AKI group; $82.5 \%$ had fluid balance monitoring, $7.5 \%$ had a urine dip, $40 \%$ had a VBG, $12.5 \%$ had a renal tract ultrasound, $57.5 \%$ had their drug charts reviewed and $7.5 \%$ were ultimately referred to a Renal Physician for further assessment. In the community AKI group, $64 \%$ had fluid balance monitoring, $75 \%$ had a urine dip, $76 \%$ had a VBG, $32 \%$ had a renal tract ultrasound, $90 \%$ had their drug charts reviewed and $5 \%$ were ultimately referred to a Renal Physician for further assessment (Figures 2 and 3).

\section{Discussion}

At a brief glance, we seem to be faring better in managing community AKI's as compared to hospital AKI's. We can also see that those above the age of 75 are at a higher risk of developing AKI. Hence, it would be prudent to involve community GP's, family members and social services to organize help at home in the form of a package of care or consideration of transfer to a care home, especially those with advanced dementia, frailty and poor physiological reserve.

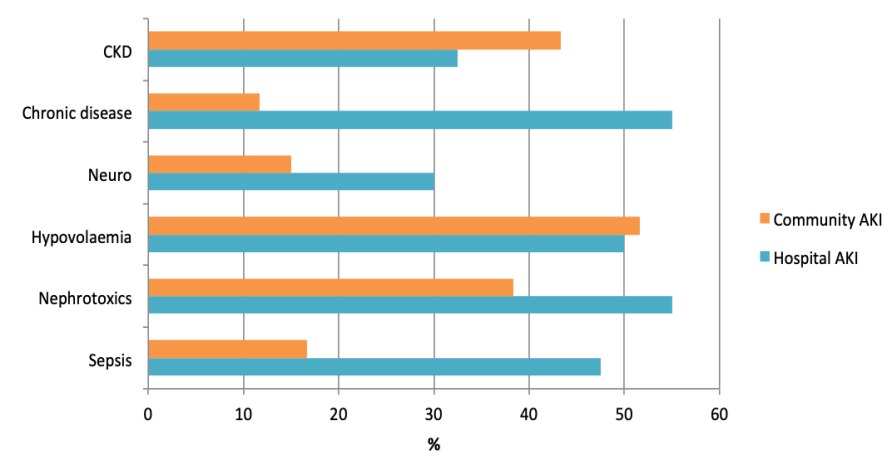

Figure 2. Risk Factors: Comparison of community AKI group vs. hospital AKI group

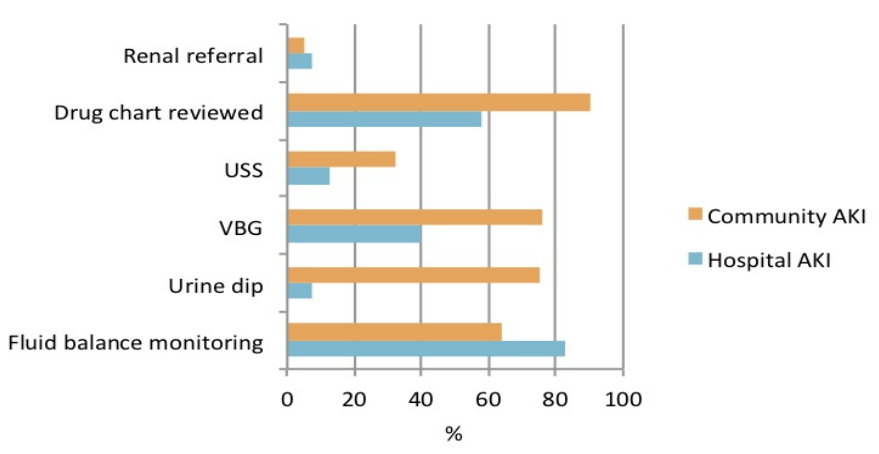

Figure 3. Management: Comparison of community AKI group vs. hospital AKI group

On admission and subsequent ward rounds, it is always important to review patient regular medications, paying particular attention to nephrotoxics that can worsen current AKI. It is also important for healthcare professionals to be aware of deteriorating NEWS score and how to best interpret them based on clinical evaluation and judgement.

As chronic diseases have a major contribution to development of AKI, it is vital for healthcare professionals to continue to emphasize lifestyle modification (exercise and healthy diet). For example, diabetics need to be reminded of the importance of optimizing their blood sugar management to minimize the risk of diabetic complications, such as CKD. 


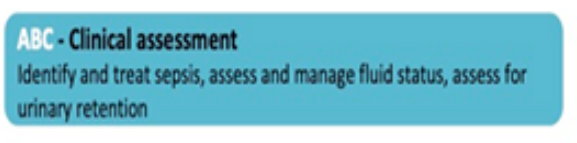

Drug chart review
Review and stop nephrotoxics, adjust dosing if needed, hold anti-
hypertensives if hypotension is present
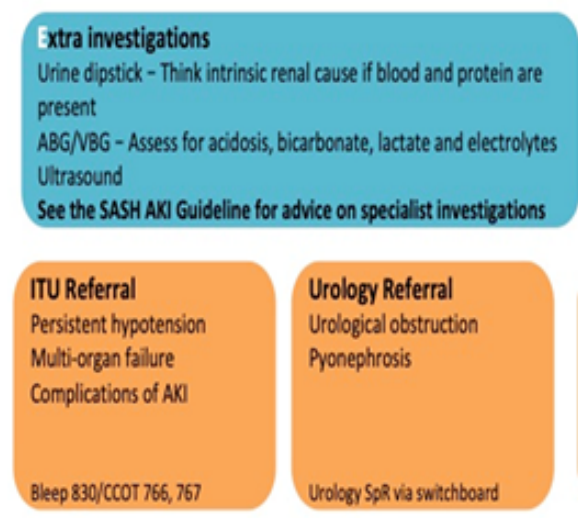

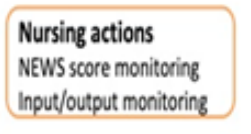

Pharmacist actions

Drug chart review and

dose adjustments

Complications of AKI
Metabolic acidosis
Refractony hyperkalemia
Pulmonary oedema
Anuria
Uraemia

Renal referral
AKI 3/Non recovering AKI
Renal transplant/CKD 4,5
Renal cause of AKI suspected
Complications of AKI
Renal SpR Ria St Heliers/Brighton

\section{Risk Assessment}

All patients should be assessed for risk factors for AKI on admission
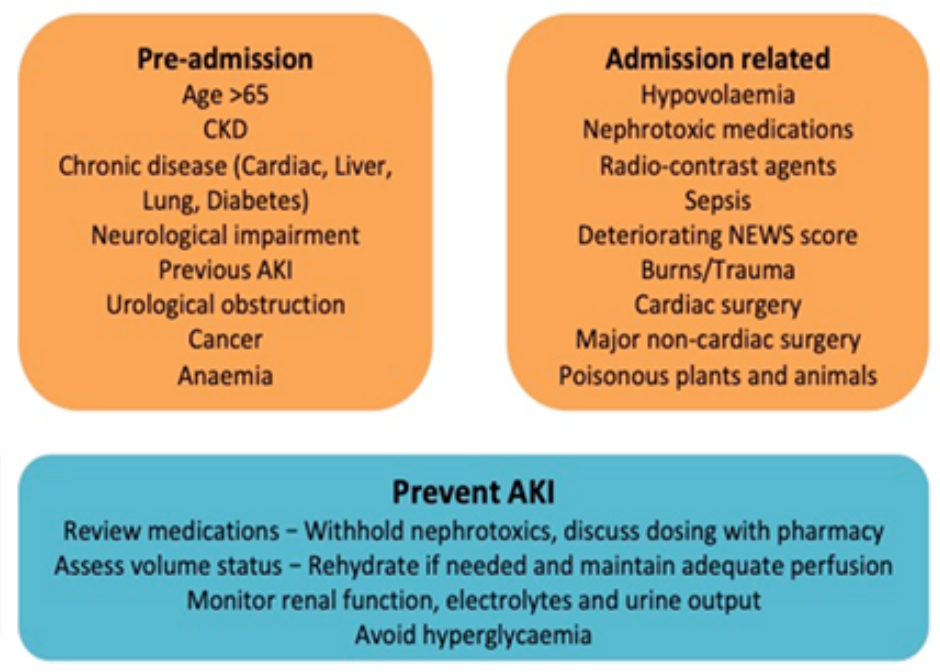

Figure 4. AKI care bundle

It should also be noted that referral to Renal Physicians of patients with AKI on background worsening CKD should be done promptly given better prognosis with early intervention with dialysis or possible renal transplant.

\section{Conclusion}

Based on the clinical audit, we can conclude that the elderly population are at higher risk of developing AKI, whether it be in the community or in hospital, thus requiring a multi-disciplinary team effort to help better identify this at risk population.

Previous AKI is an important risk factor that can be identified on admission and highlights patients at risk of developing AKI at an early stage, hence a monitoring system should be instituted to identify these patients in the community to minimize risk of hospital admissions.

This drives home the important message that the management of AKI should be standardised to optimise care of these patients. This can be done in the form of an AKI Care Bundle to improve the risk assessment, recognition, and management of this acute condition.

\section{Action plan}

Within our local population, we have identified a number of modifiable risk factors that could be addressed to prevent AKI if identified early. We have developed guidance specifically targeted at these modifiable risk factors.

Management of AKI differed according to when AKI developed. This reflects differences in current AKI management across the trust due to lack of trust specific standardised guidelines.

This data has been presented locally at departmental and medical division meetings and led to the development of a hospital AKI Guideline, with a focus on risk assessment and prevention. We are creating an AKI electronic alert to identify patients who have had a previous AKI on admission. The audit has also led to streamlining of our AKI alerting system to improve the escalation and recognition of AKI.

Posters and educational sessions have been set up to improve the risk assessment, recognition and management of AKI (Figure 4).

\section{References}

1. UK NICE (2019) Guidance on Management of AKI.

2. Basile DP, Anderson MD, Sutton TA (2012) Pathophysiology of acute kidney injury Compr Physiol 2: 1303-1353.

3. Makris K, Spanou L (2016) Acute kidney injury: Definition, pathophysiology and clinical phenotypes. Clin Biochem Rev 37: 85-98.

4. Rahman M, Shad F, Smith MC (2012) Acute kidney injury: A guide to diagnosis and management. Am Fam Physician 86: 631-639.

5. Doyle JF, Forni LG (2016) Acute kidney injury: short-term and long-term effects. Critical Care 20: 188.

Copyright: (C2020 Paranthaman KS. This is an open-access article distributed under the terms of the Creative Commons Attribution License, which permits unrestricted use, distribution, and reproduction in any medium, provided the original author and source are credited. 